\title{
Article
}

Jeffrey Sullivan, Tiffany M. Shih, Emma van Eijndhoven*, Yash J. Jalundhwala, Darius N. Lakdawalla, Cindy Zadikoff, Jennifer Benner, Thomas S. Marshall and Kavita R. Sail

\section{The Social Value of Improvement in Activities of Daily Living among the Advanced Parkinson's Disease Population}

https://doi.org/10.1515/fhep-2019-0021

Received August 1, 2020; accepted September 21, 2020

\section{Abstract}

Objectives: Quantify the value of functional status (FS) improvements consistent in magnitude with improvements due to levodopa-carbidopa intestinal gel (LCIG) treatment, among the advanced Parkinson's disease (APD) population.

Methods: The Health Economic Medical Innovation Simulation (THEMIS), a microsimulation that estimates future health conditions and medical spending, was used to quantify the health and cost burden of disability among the APD population, and the value of quality-adjusted life-years gained from FS improvement due to LCIG treatment compared to standard of care (SoC). A US-representative Parkinson's disease (PD)-comparable cohort was constructed in THEMIS based on observed PD patient characteristics in a nationally representative dataset. APD was defined from the literature and clinical expert input. The PD and APD cohorts were followed from 2010 over their remaining lifetimes. All individuals were ages 65 and over at the start of the simulation. To estimate

\footnotetext{
*Corresponding author: Emma van Eijndhoven MS, MA, PRECISIONheor, 11100 Santa Monica Boulevard, Suite 500, Los Angeles, CA 90025, USA,

E-mail: emma.vaneijndhoven@precisionvh.com

Jeffrey Sullivan, MS, Tiffany M. Shih, PhD, Darius N. Lakdawalla, PhD and Jennifer Benner, BS, PRECISIONheor, 11100 Santa Monica Boulevard, Suite 500, Los Angeles, CA 90025, USA

Yash J. Jalundhwala, PhD, Thomas S. Marshall, PharmD and Kavita R. Sail, PhD, AbbVie, Health Economics and Outcomes Research, 1 North Waukegan Rd, D-GMH1, AP31-1E, North Chicago, IL 60064, USA

Cindy Zadikoff, MD, AbbVie, Health Economics and Outcomes Research, 1 North Waukegan Rd, DGMH1, AP31-1E, North Chicago, IL 60064, USA; and Feinberg School of Medicine, Northwestern University, Abbott Hall Suite 1123, 710 N Lake Shore Drive, Chicago, IL 60611, USA
} 
the value of FS improvement due to LCIG treatment, decreases in activities of daily living (ADL) limitations caused by LCIG treatment were calculated using data from a randomized, controlled, double-blind, double-dummy clinical trial and applied to the APD population in THEMIS.

Results: Total burden of disability associated with APD was $\$ 17.7$ billion (B). From clinical trial data, LCIG treatment versus SoC lowers the odds of difficulties in walking, dressing, and bathing by $76 \%, 42 \%$ and $39 \%$, respectively. Among the APD population, these reductions generated $\$ 2.6 \mathrm{~B}$ in value to patients and cost savings to payers. The added value was $15 \%$ of the burden of disability associated with APD and offsets $15 \%$ of the cost of LCIG treatment.

Conclusions: FS improvements, consistent with improvements due to LCIG treatment, in the APD population created health benefits and reduced healthcare costs in the US.

Keywords: simulation, advanced Parkinson's disease, functional status, activities of daily living, instrumental activities of daily living

\section{Introduction}

Parkinson's Disease (PD) is a chronic neurodegenerative progressive disease characterized by motor and non-motor symptoms that impact patients' health-related quality of life (QoL) (Soh, Morris, and McGinley 2011). It is the second most common neurodegenerative disorder and its prevalence is expected to grow over coming decades in part due to the population aging (de Lau and Breteler 2006; Dorsey et al. 2007; National Institute of Neurological Disorders and Stroke 2015). Indeed, prevalence rises with age in global data: the prevalence of PD is 41 per 100,000 among those aged 40-49 years, but 1,903 per 100,000 among individuals aged 80 and older (Pringsheim et al. 2014). Similarly, PD prevalence in the US increases starting at age 55 and peaks at a rate of $1.85 \%$ among 80 year-olds (Kowal et al. 2013). Given that the elderly population is expected to double between 2000 and 2050, overall prevalence might rise even further (Congressional Budget Office 2013). Moreover, the economic and social burden of PD is substantial. Among neurological disorders globally, PD ranks within the top seven causes of disability-adjusted life years lost (World Health Organization 2007). Direct and indirect costs for PD in the US were estimated to exceed \$14.4 billion (B) in 2010 (Kowal et al. 2013).

The burden borne by patients may be particularly high among patients who suffer from more advanced PD. PD progression imposes increased costs and decreased QoL (Johnson et al. 2013a). Over time, reduced functional capabilities heighten a patient's need for increased assistance with day-to-day activities. Individuals with advanced 
Parkinson's disease (APD) experience a decline in functional status (FS), i.e., the ability to perform daily tasks in order to fulfill self-care needs and usual roles, and to preserve health and well-being (Leidy 1994; American Thoracic Society 2007; Shih et al. 2020). This decline has been shown to increase the risk of nursing home admission (NHA) among APD patients in the US (Shih et al. 2020). In addition, limitations in activities of daily living (ADLs), a commonly used measure of FS, contribute to the risk of NHA and health care costs (Chan et al. 2002; Fong, Mitchell, and Koh 2015). The total health and cost burden due to decline in FS among the APD population may therefore be substantial, but has not yet been quantified. This component of burden has become a more pressing research question with the arrival of therapies that aim to slow FS decline among APD patients.

In this study, the total burden resulting from decline in FS due to APD and the corresponding value generated by an APD treatment that reduces the risk of decline in FS among APD patients were estimated. The 'social value' of FS improvement refers to the dollar value of improvement in health and economic outcomes to all stakeholders due to FS improvement, net of all corresponding costs. Levodopa-carbidopa intestinal gel (LCIG) improves off-time and on-time without troublesome dyskinesia and hence reduces limitations in ADLs in APD patients (Standaert et al. 2013). This study estimates the value that these clinical benefits generate in reduced medical costs and improved QoL by utilizing The Health Economics Medical Innovation Simulation (THEMIS) model, used in prior work studying the social value of medical innovation and health improvement (Lakdawalla et al., 2003, 2013; Van Nuys et al. 2014), to quantify the social burden of disability due to APD and to estimate the social value of improvement in FS. Improvement in FS likely resulting from LCIG treatment of APD patients were measured and quantified as a reduction in ADL limitations.

\section{Methods}

\subsection{Study Design}

This study simulated the burden of disability due to APD and the lifetime effects of LCIG treatment on a simulated cohort of APD patients, using THEMIS, a Monte-Carlo simulation that projects health and economic outcomes for the elderly population in the US (Eber et al. 2015; Goldman et al. 2013; Lakdawalla et al., 2003, 2013; Van Nuys et al. 2014). THEMIS brings together clinical trial results, observational datasets, and econometric methods into a single framework that allows researchers to compute the value to society of various medical and social 
interventions. ${ }^{1}$ For this study, a refined THEMIS modeling framework was used that projects the lifetime trajectory of health, FS, and medical spending for US residents over age 65 with non-advanced PD or APD.

Figure 1 illustrates the study design. First, the PD-comparable and APD cohorts were defined and exposed to THEMIS health transition equations. Two separate interventions were applied to determine (1) the burden of disability due to APD, and (2) the effects of LCIG treatment relative to the standard of care (SoC). The total burden of disability due to APD was estimated in THEMIS. Since this burden is equivalent to the value of eliminating disability due to APD, the burden was simulated as the incremental social value gained when ADLs in the APD population fall to the levels seen in the non-advanced PD population. The value of improved FS from LCIG treatment was estimated as the incremental social value that results from improving ADLs to the degree suggested by a randomized, controlled, double-blind, double-dummy clinical trial that compares LCIG treatment to the SoC (optimized oral levodopa-carbidopa IR) in the APD population (Olanow et al. 2014). The endpoints used to measure social value were qualityadjusted life-years (QALYs) and total medical spending. The PD and APD cohorts were followed starting in 2010 over their remaining lifetimes. A more detailed flowchart of the study design can be found in the Appendix Figure 1.

\subsection{Data Sources}

The 1998-2010 Health and Retirement Study (HRS) data were used to construct the population for the analysis and estimate disease, FS, and mortality transition models within THEMIS. The HRS is a nationally representative biannual longitudinal panel survey of US residents over age 50 . There is no specific identifier for PD status in the HRS data or, to our knowledge, any other nationally representative lifetime panel study of the elderly population. Therefore, a PD-comparable cohort was constructed in the HRS by using the Medicare Current Beneficiary Survey (MCBS) Cost and Use files (1992-2010). The MCBS is a nationally representative rotating panel of aged (65 and older), disabled, and institutionalized individuals within Medicare. The MCBS follows respondents for up to 4 years and annually refreshes with new survey respondents to maintain national representativeness over time. The MCBS data is a combination of self-reported survey data and claims data. PD was identified through self-reports using the question: "[[Since (LAST HF MONTH YEAR) has/Has] a doctor (ever) told [you/(SP)] that

1 An additional flowchart of the study design can be found in the Appendix. More information on THEMIS is provided in THEMIS HRS technical documentation (PRECISIONheor 2019). 


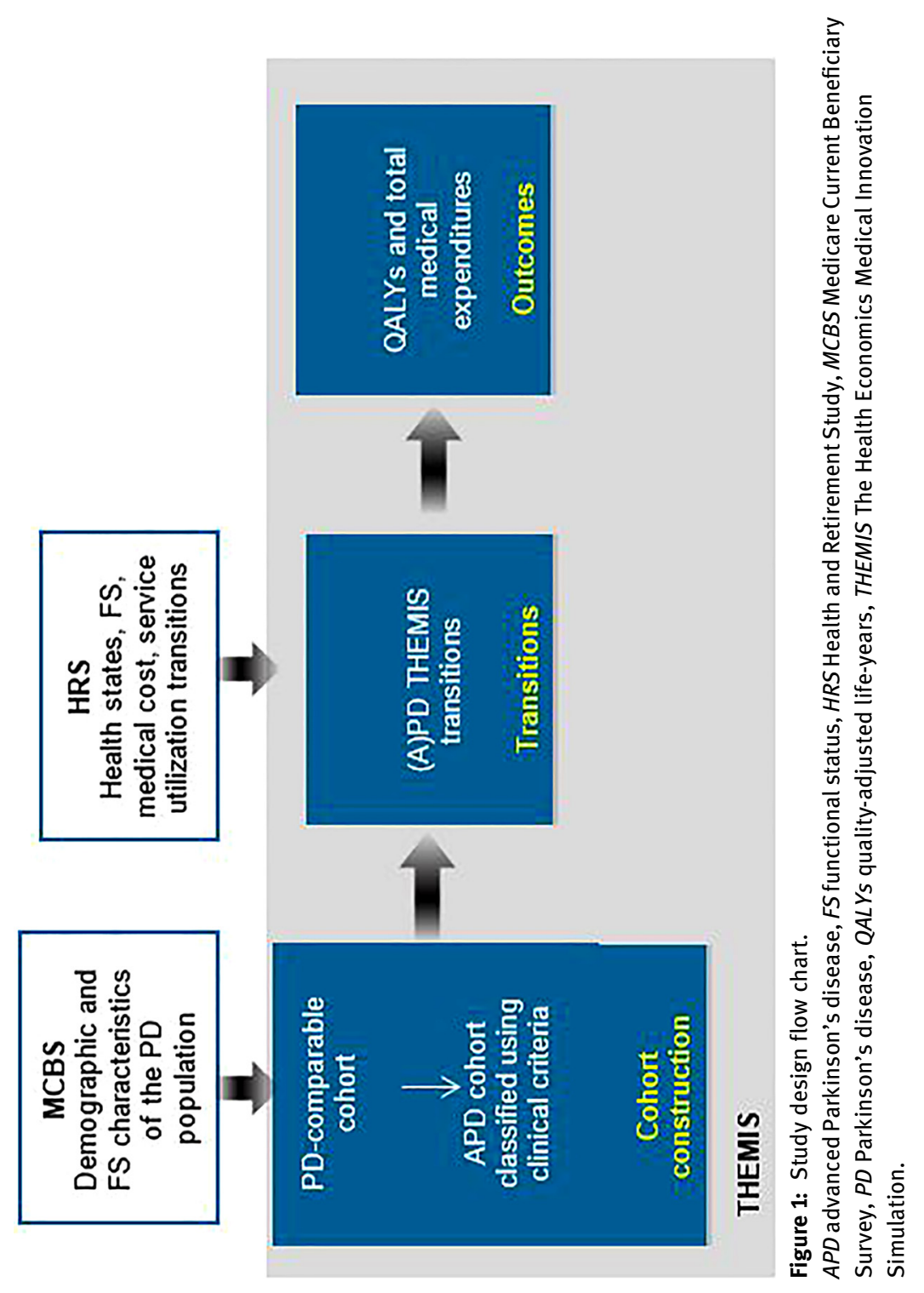


(you/he/she) had ... I Parkinson's disease?” In addition, the MCBS was used to estimate total medical expenditures (i.e., medical and pharmacy cost) for individuals aged 65 and older, as the MCBS has administrative cost data that is more reliable than the self-reported data in the HRS (Goldman et al. 2013).

The Medicare Expenditure Panel Survey (MEPS) data were used to estimate utility, measured by EQ-5D, for all ages in each year of life as a function of comorbidities (Goldman et al. 2013; van Reenen and Janssen 2015). The MEPS is an annual nationally representative rotating panel survey of families and individuals, their medical providers (doctors, hospitals, pharmacies, etc.), and employers across the US.

Finally, data from a Phase 3, 12-week, randomized, double-blind, doubledummy, clinical trial (control: optimized oral levodopa-carbidopa IR) were used to estimate the effect of LCIG use on the risk of having limitations in ADLs (Olanow et al. 2014), The trial evaluated the efficacy, safety, and tolerability of LCIG in levodopa-responsive APD subjects who, despite receiving optimal oral treatments for PD, continued to experience persistent motor fluctuations. Results of the Unified Parkinson's Disease Rating Scale (UPDRS) Part II, a secondary trial endpoint, were used to estimate the impact of LCIG treatment on ADLs.

\subsection{Cohort Construction}

As PD status is not reported in the HRS, the HRS sample was reweighted to match the distribution of demographics (sex, race, and age) and whether an individual is living in a nursing home of the PD patients in the MCBS in order to create a "PD-comparable cohort". Specifically, means of sex, race, age, and whether an individual is living in a nursing home were generated using the weighted MCBS data, and then used to reweight the population weights in the HRS. Thus, creating a HRS sample with a similar demographic composition as the PD sample in the MCBS.

Based on literature and clinical input (Antonini et al. 2015), APD patients were defined as PD patients that experienced repeated falls (more than 3 ) or were unable to walk unassisted in the prior year. Two methods were used to identify APD patients according to this definition. The baseline estimation began by first generating the PD-comparable cohort in the HRS (see description above). APD patients were identified within the PD-comparable cohort using the HRS selfreported identifiers for repeated falls or prior use of an ambulatory assistance device, namely "How many times have you fallen [[/since [PREV WAVE FIRST R IW MO], [PREV WAVE FIRST R IW YEAR]/since [PREV WAVE FIRST R IW YEAR]/in the last two years]]/in the last two years]?"; and “Do you ever use equipment or devices such as a cane, walker or wheelchair when crossing a room?” In a sensitivity 
analysis, both PD and APD patients were first identified in the MCBS. APD status was identified through self-reports using the questions "Since (LAST HF MONTH YEAR), how many times [have you/has (SP)] fallen down?"; and “[Do you/Does (SP)] use special equipment or aids to help (you/him/her) with walking?” For this sensitivity analysis, a model of the probability of APD was estimated in the MCBS as a function of age, gender, race, ethnicity, widowhood, body mass index, smoking status, limitations in ADLs, and limitations in instrumental activities of daily living (IADLs) (see Appendix Table 1). The model was then applied to the HRS population to recover the probability of having APD among HRS respondents. This probability was used to identify likely APD patients using Monte Carlo methods -a random number between zero and one was drawn, and if the number drawn fell below the estimated probability of APD, a patient was classified as having APD. The baseline strategy for identifying APD was preferred as it was more direct, i.e., native data was used in modeling APD, rather than solely relying on an imputation framework. Additionally, this strategy enabled us to study the PD population as a whole rather than just the APD population.

\subsection{Estimating Transitions}

THEMIS consists of biennial transition models that predict the onset of health and disability states (i.e., cancer, heart disease, lung disease, diabetes, stroke, and functional status), and economic outcomes (i.e., total medical expenditures and health service utilization). The transition models for functional status include limitations in any ADL, limitations in any IADL, and for each specific ADL and IADL limitations. The ADLs included walking, eating, dressing, bathing, toileting, and getting out of bed/chair; the IADLs included are using the telephone, taking medications, and handling money. The probability of experiencing difficulties with some particular ADLs (walking, dressing, and bathing) for individuals receiving LCIG treatment was adjusted in order to estimate the lifetime effects of LCIG treatment (more details in Section 2.5.2). Predictions for the next period (two years from the current period) were based on current period health, disability, economic, and demographic states. These predictions were derived from transition models that were estimated for a nationally representative sample of individuals aged 51 and older in the HRS data and have been detailed in prior studies (Goldman et al. 2013). The result of estimating these transitions was a lifetime profile of comorbidities and FS for each respondent. In the sensitivity analysis, transition models predicting NHAs and medical costs among non-advanced PD and APD patients were also estimated. These transition models were estimated using SAS and STATA, and included in the Online Appendix. 
FS was used as a predictor in several transition models (see Online Appendix). Limitations in individual ADLs (walking, eating, dressing, bathing, toileting, and getting out of bed/chair) and IADLs (using the telephone, taking medications, and handling money) in the current period affect mortality, memory problems, cognitive status, depressive symptoms, smoking, caregiver burden (hours), BMI, pain status, and nursing home living in the next period (2 years). They also affect the probability of experiencing limitations in any ADLs, limitations in any IADLs, limitations in individual ADLs, and limitations in individual IADLs in the next period. In addition, having three or more ADLs in the current period affects Medicaid eligibility, and healthcare expenditures and utilization. Number of ADLs and IADs also effects QALYs in the current period.

\subsection{Interventions}

\subsubsection{Elimination of Disability due to APD}

To provide context for the value of disability reduction achieved through LCIG treatment, the total burden of disability due to APD was estimated. Specifically, THEMIS transition models were first applied to the PD-comparable and APD cohorts in the HRS starting in 2010 in order to simulate over the individuals' remaining lifetime in the baseline scenario. This baseline scenario was then compared to a scenario in which APD patients had the same level of disability as seen in the PD population. For each APD patient in the simulation, the predicted probability of each disability limitation was reduced by a uniform factor set equal to the percent difference in disability between the APD and PD populations. The result of this adjustment was a simulation in which disability rates were equal across the APD and PD populations. This allowed us to estimate the social burden of incremental disability imposed by APD.

\subsubsection{Effects of LCIG Treatment on Disability Reduction}

In order to incorporate the effect of LCIG treatment on FS limitations in THEMIS, the probability of experiencing difficulties in some ADLs for individuals receiving LCIG treatment was adjusted to reflect the effects observed in the clinical trial. First, disaggregated clinical trial data was used to calculate the odds ratio of difficulty for individuals receiving LCIG treatment relative to the odds ratio of difficulty for individuals not receiving treatment: 
$r=\frac{\left(\frac{\text { Proportion of subjects with limitation, } L C I G \text { treatment }}{\text { Proportion of subjects without liaitatition, LCII treatment }}\right)}{\left(\frac{\text { Proportion of subjects with limitation, SoC }}{\text { Proportion of subjects without limitation, SoC }}\right)}$. As only walking, dressing, and bathing were available in both the trial and the HRS data, our analysis only incorporated the effect of LCIG treatment on limitations in these ADLs. The disaggregated clinical trial results for these activities were identified from the UPDRS Part II score. Second, the resulting odds ratios for the three ADLs were applied among APD patients in the simulation by adjusting the probability of experiencing difficulty with a particular ADL for individuals receiving LCIG treatment to $p_{1}=\frac{r \times p_{2}}{1+r \times p_{2}-p_{2}}$, where $p_{2}$ is the baseline probability of experiencing difficulty with a particular ADL, and $r$ is the odds ratio. Because providers may not utilize LCIG treatment for those with dementia, individuals with dementia were assumed to not receive LCIG treatment.

\subsection{Endpoints}

The primary endpoints were quality-adjusted life years (QALYs) gained by patients due to FS improvement, and total medical expenditures across payers and patients over the remaining lifetime of the PD and APD cohorts starting in 2010. QALYs were calculated using utility, measured by the EQ-5D (van Reenen and Janssen 2015). MEPS data yielded estimates of utility as a function of health and number of ADL limitations. These estimates were then applied to HRS data on health and FS to measure the corresponding utility for each respondent (Goldman et al. 2013; van Reenen and Janssen 2015). Total medical expenditures were calculated using all medical payments made for a patient by all payers. Additional related endpoints include Medicaid enrollment and spending, and NHAs.

The social value of an intervention affecting FS was estimated as the value of QALYs gained, net of additional medical expenditures across payers and patients, resulting from the intervention, and was relative to the baseline scenario. In THEMIS, estimated social value incorporates, among other components, the value of improvement in QALYs, changes in disease incidence and prevalence, and changes in healthcare costs (broken down by type and payer). Specifically, value was defined as $V=\sum_{t=2010}^{n} \sum_{i}\left(Q_{i, t}^{\prime}-Q_{i, t}\right)-\left(M_{i, t}^{\prime}-M_{i, t}\right)-\left(G_{i, t}^{\prime}-G_{i, t}\right)$ where $V$ is the total social value for each individual $i$ in the PD and APD cohorts over the individual's lifetime, $Q_{i, t}$ is the value of a QALY for individual $i$ in year $t, M_{i, t}$ are non-government medical expenditures (e.g., out-of-pocket payments) for the benefit of individual $i$ in year $t$, and $G_{i, t}$ are other government expenditures for the benefit of individual $i$ in year $t$ (e.g., social security payments, Medicare and Medicaid payments, supplemental security income, and other government 
transfers). $Q_{i, t}^{\prime}$ is the QALY level under the intervention while $Q_{i, t}$ is under the baseline scenario, similarly for $M$ and $G$. Total medical expenditures were therefore given by $M+G$. Based on estimates from the literature, each QALY was valued at $\$ 200,000$ (Viscusi and Aldy 2003). In a sensitivity analysis, an alternative QALY value of $\$ 50,000$ was utilized (Neumann, Cohen, and Weinstein 2014).

It is not strictly appropriate to compare the total cost of LCIG treatment to the estimated social value of FS improvement from LCIG, because the calculation of value from treatment was limited to FS improvement only. Nonetheless, it is helpful to consider costs alongside this subset of LCIG's value. As a point of comparison, total costs of LCIG treatment for the APD population were estimated. Treatment cost was based on the total LCIG treatment years of the APD population and the annual wholesale acquisition cost of \$73,470 in 2015 (Truven Health Analytics Microdex Solutions 2016). ${ }^{2}$ In this and all other cases, dollar values for each endpoint were inflated to $2015 \$$.

\section{Results}

\subsection{Descriptive Analysis}

Table 1 provides the summary statistics for the baseline year (2010) of the simulation. In the HRS, approximately $11 \%$ of the PD population was classified as having APD (57,029 patients in 2010) according to the weighted population numbers. Individuals in the HRS APD cohort were more likely to experience difficulty with any given ADL and IADL ( $p<0.001$ for all), had difficulty with more ADLs and IADLs on average ( $p<0.001$ for both), had higher rates of comorbidities ( $p<0.001$ for all), and were more likely to have prior NHAs than individuals with non-advanced PD $(p<0.001)$.

\subsection{Simulation Results}

\subsubsection{Burden of Disability due to APD}

As shown in Figure 2, alleviating disability due to APD increased QALYs and monetized QALYs by 89,858 and $\$ 18.0 B$, respectively, but also increased medical expenditures by $\$ 0.3 \mathrm{~B}$ due to an increase of 195,946 patient life-years, relative to a

2 The wholesale acquisition cost includes the annual cost of the treatment but does not include the cost of the procedure to implant the device. 


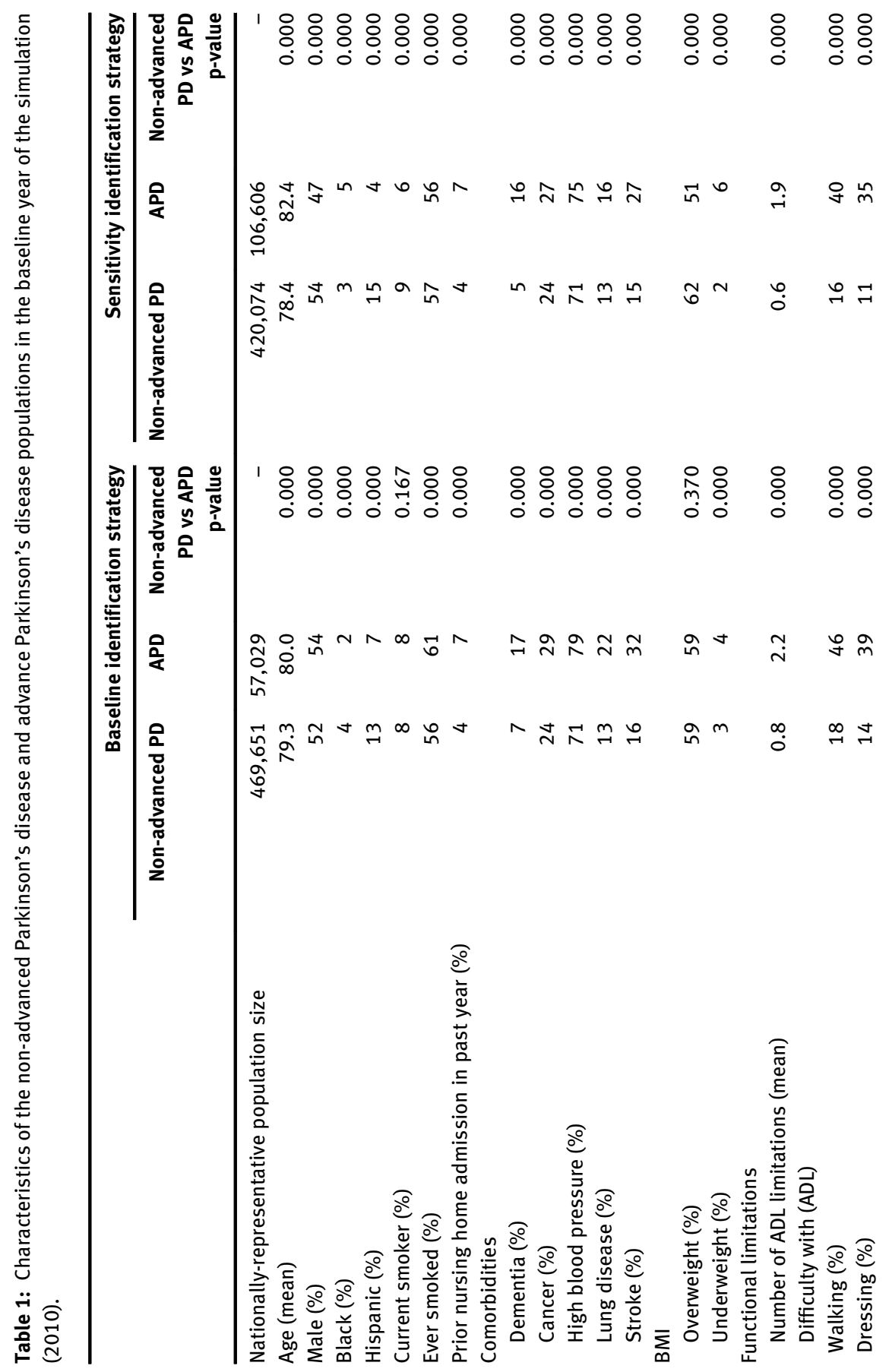




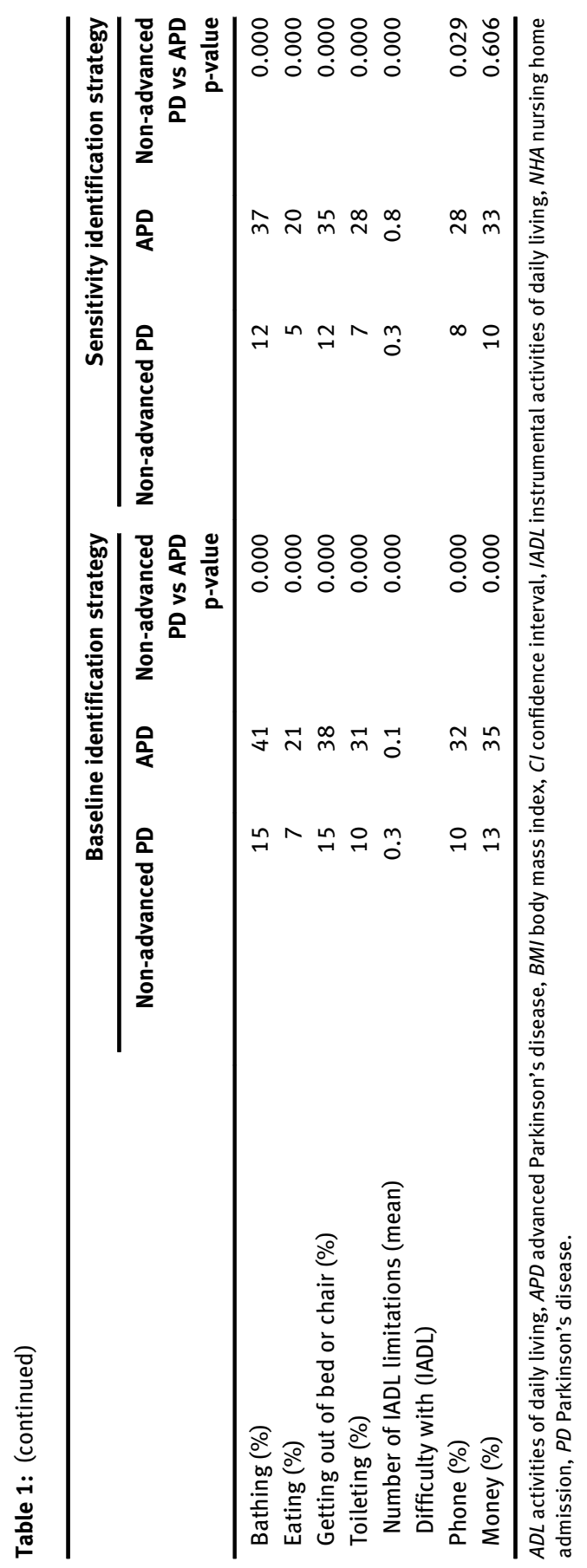




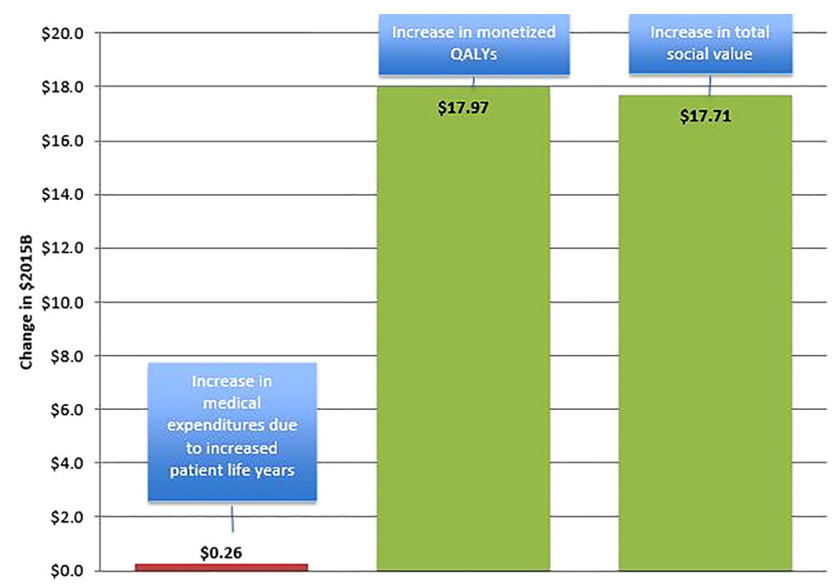

Figure 2: Change in outcomes from reducing disability due to APD.

$B$ billion, $Q A L Y$ quality-adjusted life year.

baseline of 8,381,166 patient life-years. The total social burden of alleviating disability due to APD was $\$ 17.7 \mathrm{~B}$.

\subsubsection{Value of Improvement in Limitations of ADLs due to LCIG Treatment}

Figure 3 provides the effects of LCIG treatment on walking, dressing, and bathing, as estimated from the clinical trial data (Olanow et al. 2014). The effects of LCIG treatment compared to SoC lowered the odds of difficulty in walking, dressing, and bathing by $76 \%, 43 \%$ and $38 \%$, respectively. Table 2 provides the health and cost outcomes resulting from LCIG treatment under the baseline and sensitivity scenarios. When applied simultaneously, reductions in difficulty with walking, dressing, and bathing due to LCIG treatment in the APD cohort generated 15,946 patient life-years, 10,631 QALYs (equivalent to $8.1 \%$ of the total life-year burden and $11.8 \%$ of the total QALY burden associated with disability due to by APD), and $\$ 0.5 \mathrm{~B}$ in savings in medical expenditures by all payers over the lifetime of all APD patients, relative to SoC. The value of health improvement and cost savings from improving all three ADLs due to LCIG treatment totaled $\$ 2.6 \mathrm{~B}$ in value across all payers and patients relative to SoC. This value was equivalent to $15 \%$ of the total disability burden of APD and offset $15 \%$ of LCIG treatment costs among the APD population. Medicaid spending decreased by $\$ 0.2 \mathrm{~B}$, partially due to 3692 fewer patient-years spent by LCIG treated APD patients in nursing homes and 7450 fewer person-years of Medicaid enrollment. 


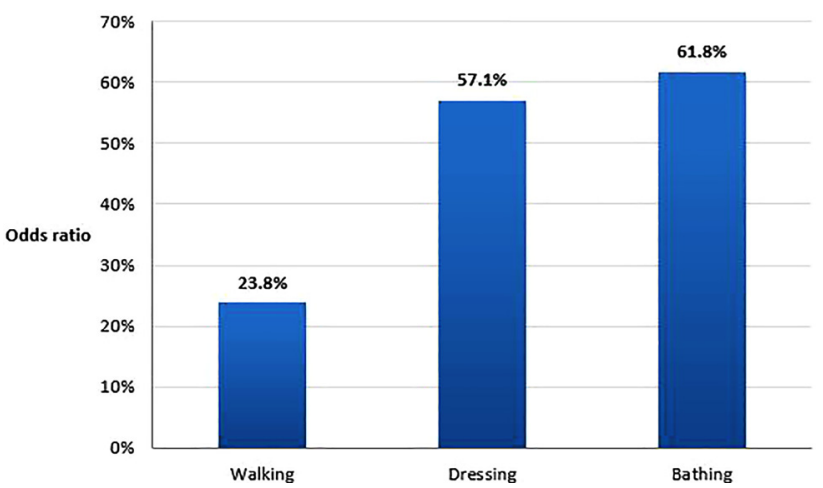

Figure 3: Odds ratio of experiencing FS limitations for individuals treated with LCIG versus individuals under SoC, based on 12-week clinical trial results.

FS functional status, LCIG levodopa-carbidopa intestinal gel, SoC standard of care

The clinical trial UPDRS Part II secondary endpoint results were disaggregated and then used to estimate the proportion of subjects with difficulty in each ADL, by treatment arm (Olanow et al. 2014). Specifically, for each ADL, the odds ratio of difficulty for individuals receiving LCIG treatment versus individuals receiving $\mathrm{SoC}$ was calculated using the 12-week trial results as follows:

$$
\text { Odds ratio }=\frac{\left(\frac{\text { Proportion of subjects with limitation, LCIG treatment }}{\text { Proportion of subjects without limitation, LCIG treatment }}\right)}{\left(\frac{\text { Proportion of subjects with limitation, SoC }}{\text { Proportion of subjects without limitation, SoC }}\right)} .
$$

\subsubsection{Value of Individual Improvement in Limitations of ADLs}

As shown in Table 2, the social values associated with improvement in walking, dressing, and bathing for the APD population due to LCIG treatment compared to SoC were $\$ 1.4 \mathrm{~B}, \$ 0.9 \mathrm{~B}$, and $\$ 0.2 \mathrm{~B}$, respectively. The value of improvement in each individual ADL was less than the social value derived from simultaneously improving all three ADLs. Improvement in walking led to the largest reduction in total medical expenditures, as well as to Medicaid expenditures, nursing home patient-years, and Medicaid enrollment person-years. Improvement in dressing limitations was associated with the largest improvement in patient life-years and QALYs. However, in this case, patient life-years increased far more than QALYs, indicating that the QoL for these additional life-years was relatively low.

\subsection{Sensitivity Analyses}

The baseline classification of APD was repeated falls or use of an ambulatory assistance device as measured in the HRS within the PD-comparable cohort. However, using the alternative classification of APD (imputing APD status in the HRS based on the characteristics of the APD population in the MCBS), the APD 


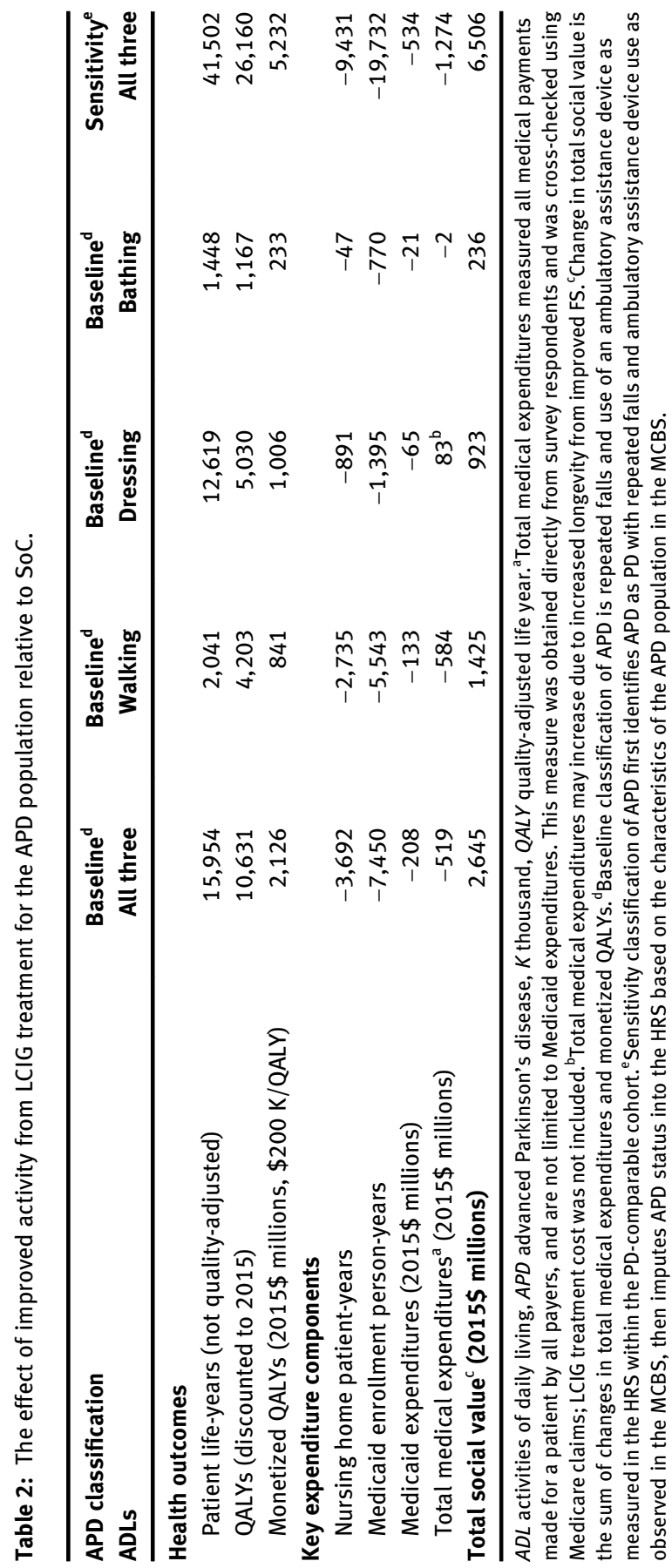


population constituted $20 \%(106,606)$ of the PD population according to the weighted population size (Table 1). The burden of disability due to APD was estimated to be $\$ 51.5 \mathrm{~B}$, incorporating a loss of 643,320 patient life-years and 266,805 QALYs. Improved walking, dressing, and bathing from LCIG treatment provided a value of $\$ 6.5 \mathrm{~B}$, or approximately $13 \%$ the burden of APD (Table 2). This value also offsets $14 \%$ of the LCIG treatment cost incurred by the APD population.

A second sensitivity analysis employed a lower value of \$50,000 per QALY. For this sensitivity analysis, the total burden of APD and the value of FS improvement due to LCIG treatment came to $\$ 5.1 \mathrm{~B}$ and $\$ 1.2 \mathrm{~B}$, respectively. The improvement to walking, dressing, and bathing through LCIG treatment offset $13 \%$ of the disability burden associated with APD.

\section{Discussion}

This study estimated the value of FS improvements that are consistent in magnitude with improvements due to LCIG treatment, over the lifetime of the APD population, using a microsimulation model that predicts long-term economic and health outcomes based on longitudinal transitions in the current population. Results from a randomized, controlled, double-blind, double-dummy clinical trial were leveraged to quantify the effect of LCIG treatment on FS (Olanow et al. 2014). Because the model was parameterized using nationally representative data, the results of the study are generalizable to the broader APD population in the US.

Prior work has shown that the costs of PD are substantial, particularly in the advanced stages. Johnson et al. (2013b) found that in the year following institutionalization or the adoption of an ambulatory assistance device, privately insured PD patients had 6-7 times higher healthcare costs than their non-PD counterparts (Johnson et al. (2013b)). Similarly, Kaltenboeck et al. (2012) showed that Medicare beneficiaries with early and advanced PD experienced higher direct medical costs and mortality than their non-PD counterparts (Kaltenboeck et al. 2012). The current study builds upon this prior work, but also provides new findings by (1) isolating the burden of disability in APD, (2) incorporating impacts on QoL in addition to medical expenditures, and mortality to estimate overall value, and (3) providing value estimates aggregated over a lifetime horizon. The results demonstrated that the disability component of APD alone impose a sizeable economic burden to society, equivalent to $\$ 17.7 \mathrm{~B}$.

This study also found that LCIG treatment can reduce this burden by improving FS. The combined reductions in difficulty in walking, dressing, and bathing due to LCIG treatment generated a total lifetime value of $\$ 2.6 \mathrm{~B}$ for the current APD population in the baseline estimates. Overall, improvement in these three activities reduced the total APD disability burden by $15 \%$. The impact on 
monetized QALYs due to improvement in FS from LCIG treatment constituted 80\% of the overall social value. Reduced total medical expenditures comprised the remaining $20 \%$ of the value from improved FS due to LCIG treatment. To characterize contributors to total medical expenditures, impacts of LCIG treatment on nursing home patient-years and Medicaid enrollment and expenditures were estimated over the patients' lifetimes. The probability of nursing home and Medicaid enrollment both increase for those with three or more ADL limitations. The FS improvement from LCIG treatment therefore decreased total time spent in nursing homes and Medicaid expenditures.

Furthermore, the individual components of value from LCIG treatment were identified. In particular, in the clinical trial results, LCIG treatment had the greatest impact on walking difficulty. As a result, the effect of LCIG treatment on reducing walking difficulty led to the greatest increase in social value among the three individual ADLs analyzed in this study. Improvement in walking and dressing led to similar gains in QALYs, while bathing led to smaller QALY gains. A comparison of the impact on patient life-years versus the impact on QALYs reveals that improvement in walking primarily resulted in improvement in QoL, while improvement in dressing was primarily associated with increases in patient life-years. Since dressing extended life in a relatively poor health states, it also resulted in higher medical spending. As a result of these factors, the overall value from improved dressing was lower than the value from improved walking. The analysis of individual ADLs thereby revealed that walking improvement were the key driver of the value of LCIG treatment.

There are some limitations to this study. First, PD status could not be directly observed in the HRS data. As a result, a PD cohort was imputed based on characteristics (demographics and ADLs) of the observed PD population in the MCBS. This imputation may not perfectly identify PD patients, thus creating error in our estimates. A sensitivity analysis was performed defining APD directly within the observed PD population in the MCBS data, and APD status was imputed into the HRS data to be utilized in THEMIS. Relative to the baseline results, the absolute burden of disability due to APD and value of improved FS due to LCIG treatment both increased in the sensitivity analysis. However, the proportion of the APD burden alleviated by LCIG treatment decreased slightly, from 15 to $13 \%$.

Second, the FS questionnaire in the clinical trial differed from that used in the HRS data. As a result, a restricted set of ADLs was included in the analysis, which may omit important FS determinants of value. For example, prior research has found that difficulty eating may also be influential in outcomes for the elderly population (Fong, Mitchell, and Koh 2015). Furthermore, we were only able to capture binary changes between any difficulty versus no difficulty in ADLs in THEMIS, so that actual LCIG treatment may lead to additional benefits from reductions in the severity of difficulty.

Third, a challenge was to properly define APD in order to estimate social value for this population. While there is no single definition of APD agreed upon by 
clinicians, for this study the definition of having both repeated falls and being unable to walk unassisted was used, based on clinical expert input and the results of a Delphi panel of movement disorder specialists, which was conducted to identify primary characteristics of APD patients (Antonini et al. 2015). However, estimates of the value of improved FS within the APD population may change under an alternative definition of APD.

Fourth, ADL limitations affect multiple health outcomes beyond the endpoints in this study. ADL limitations were included in the transition models of different health outcomes when the literature showed them to be a predictor of the health outcome. However, this correlation between ADL limitations and health outcomes might be due to unobserved factors. As the value of LCIG treatment is measured through its effect on ADL limitations, these other associations might lead to an overestimation of the social value of LCIG treatment.

Lastly, the current version of THEMIS does not yet have the capability to compute confidence intervals around simulation estimates. There are many sources of uncertainty in the model, since many of the transition models are based on regression estimates. This means we are unable translate the variation around a particular parameter directly into variation of the output.

\section{Conclusion}

This study found that disability due to APD imposes a substantial economic burden on society through its effects on lifespan, QoL, and medical expenditures among the APD population. Reduced limitations in FS that are of a magnitude consistent with LCIG treatment, improved health outcomes and reduced healthcare costs for APD patients in the US over their lifetimes. Future research should consider the comprehensive effect of APD treatments in reducing NHA risk and social value, incorporating its effects on a broader set of ADLs and IADLs, and should perform further sensitivity analyses around the definition of APD.

Acknowledgments: We would like to thank Suepattra May-Slater, employee of PRECISIONheor, and Warren Stevens and Shalak Gunjal, former employees of PRECISIONheor, for their support of this study. Financial support for their services was provided by AbbVie.

Declaration of funding: This study and manuscript were funded by AbbVie. The design, study conduct, and financial support for the study were provided by AbbVie. AbbVie participated in the study design, research, interpretation of data, writing, reviewing, and approving the manuscript. No honoraria or payments were made for authorship.

Declaration of financial/other relationships: Kavita Sail and Yash J. Jalundhwala are employees of AbbVie and may own AbbVie stock or stock options. Thomas 
Marshall was an employee of AbbVie at the time of the study and may own AbbVie stock or stock options. Jeffrey Sullivan and Emma van Eijndhoven are employees of PRECISIONheor. Jennifer Benner and Tiffany Shih were employees of PRECISIONheor at the time of the study. PRECISIONheor is part of Precision for Medicine Group, which receives compensation from various biopharmaceutical companies, including AbbVie. Darius N. Lakdawalla holds equity in Precision Medicine Group. Cindy Zadikoff currently is an employee of AbbVie and may own AbbVie stock or stock options. When the study was being conducted, Cindy Zadikoff was affiliated with Feinberg School of Medicine, Northwestern University, Chicago, Illinois, United States and has previously received honoraria for consulting and lecturing from AbbVie. In addition, she has received consulting honoraria from various biopharmaceutical companies.

\section{Appendix}

Appendix Table 1: Advanced Parkinson's disease model in the MCBS used to impute into the HRS (sensitivity identification strategy).

\begin{tabular}{lrr}
\hline & Coefficient & SE \\
\hline Race & & \\
Black & 0.282 & 0.230 \\
Hispanic & $-0.585^{\star \star}$ & 0.264 \\
Education & & \\
Less than high school & -0.085 & 0.150 \\
Some college and above & 0.097 & 0.155 \\
Male & 0.008 & 0.133 \\
Functional status & & \\
Two-year lag of difficulty with walking & $0.489^{\star \star \star}$ & 0.151 \\
Two-year lag of difficulty with dressing & 0.136 & 0.183 \\
Two-year lag of difficulty getting out of bed and a chair & 0.195 & 0.167 \\
Two-year lag of difficulty using the telephone & 0.116 & 0.164 \\
Two-year lag of difficulty handling money & $0.286 *$ & 0.164 \\
Two-year lag of difficulty using the toilet & 0.248 & 0.210 \\
Two-year lag of currently smoking & -0.115 & 0.248 \\
Two-year lag of being widowed & -0.027 & 0.147 \\
BMI & & \\
Two-year lag of underweight: bmi $<18.5 \mathrm{~kg} / \mathrm{m} 2$ & 0.419 & 0.536 \\
Two-year lag of overweight: bmi $25-30 \mathrm{~kg} / \mathrm{m} 2$ & -0.210 & 0.140 \\
Two-year lag of obese: bmi $\geq 30 \mathrm{~kg} / \mathrm{m} 2$ & -0.038 & 0.171 \\
Age & & \\
Two-year lag of ages 65 and-younger & -0.008 & 0.017 \\
Two-year lag of ages $65-74$ & 0.036 & 0.027 \\
Two-year lag of ages 65 and older & 0.013 & 0.014 \\
Constant & -0.710 & 1.015 \\
\hline BM body mass index, SE standad & &
\end{tabular}

$B M I$ body mass index, $S E$ standard error. 


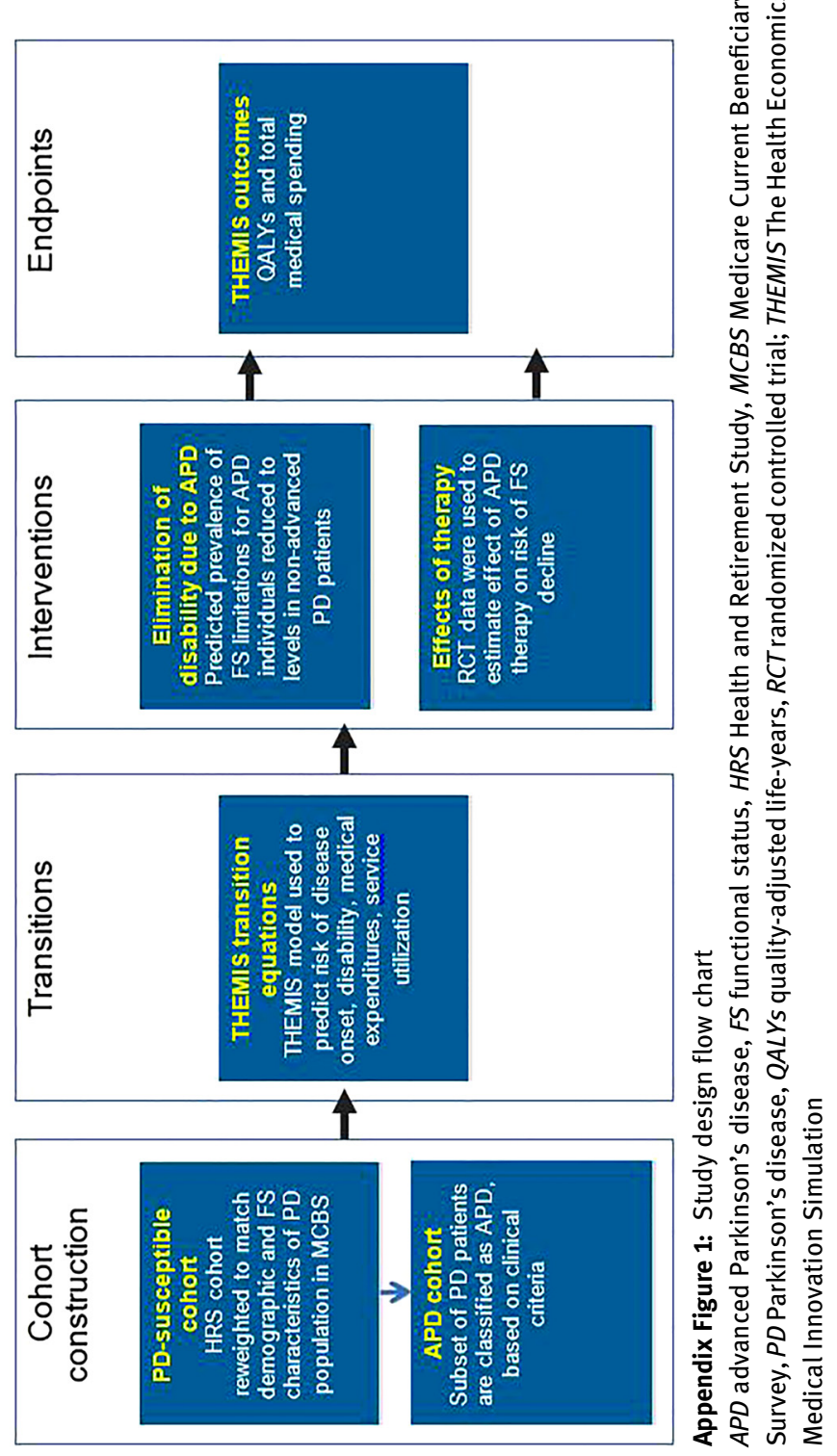




\section{References}

American Thoracic Society. 2007. "Functional Status." American Thoracic Society. Also available at https://qol.thoracic.org/sections/key-concepts/functional-status.html (accessed September 2).

Antonini, A., O. Per, L. Kleinman, A. Skalicky, T. Marshall, K. Sail, and K. Onuk. 2015. “Implementing a Delphi Panel to Improve Understanding of Patient Characteristics of Advanced Parkinson's Disease [abstract]." In Implementing a Delphi Panel to improve understanding of patient characteristics of Advanced Parkinson's disease, San Diego, CA, June 14-18, 2015.

Chan, L., S. Beaver, R. F. MacLehose, A. Jha, M. Maciejewski, and J. N. Doctor. 2002. "Disability and Health Care Costs in the Medicare Population." Archives of Physical Medicine and Rehabilitation 83 (9): 1196-201.

Congressional Budget Office. 2013. "Rising Demand for Long-Term Services and Supports for Elderly People." Congressional Budget Office. Also available at https://www.cbo.gov/sites/ default/files/113th-congress-2013-2014/reports/44363-LTC.pdf (accessed December 11).

de Lau, L. M., and M. M. Breteler. 2006. "Epidemiology of Parkinson's Disease." The Lancet Neurology 5 (6): 525-35.

Dorsey, E. R., R. Constantinescu, J. P. Thompson, K. M. Biglan, R. G. Holloway, K. Kieburtz, F. J. Marshall, B. M. Ravina, G. Schifitto, A. Siderowf, and C. M. Tanner. 2007. "Projected Number of People with Parkinson Disease in the Most Populous Nations, 2005 through 2030." Neurology 68 (5): 384-6.

Eber, M. R., D. P. Goldman, D. N. Lakdawalla, T. J. Philipson, D. Pritchard, M. Huesch, N. Summers, M. T. Linthicum, J. Sullivan, and R. W. Dubois. 2015. "Clinical Evidence Inputs to Comparative Effectiveness Research Could Impact the Development of Novel Treatments.” Journal of Comparative Effectiveness Research 7: 1-11.

Fong, J. H., O. S. Mitchell, and B. S. Koh. 2015. "Disaggregating Activities of Daily Living Limitations for Predicting Nursing Home Admission." Health Services Research 50 (2): 560-78.

Goldman, D. P., D. Cutler, J. W. Rowe, P. C. Michaud, J. Sullivan, D. Peneva, and S. J. Olshansky. 2013. "Substantial Health and Economic Returns from Delayed Aging may Warrant a New Focus for Medical Research." Health Affairs 32 (10): 1698-705.

Johnson, S. J., M. D. Diener, A. Kaltenboeck, H. G. Birnbaum, and A. D. Siderowf. 2013a. “An Economic Model of Parkinson's Disease: Implications for Slowing Progression in the United States." Movement Disorders 28 (3): 319-26.

Johnson, S. J., K. Anna, M. Diener, H. G. Birnbaum, E. Grubb, J. Castelli-Haley, and A. D. Siderowf. 2013b. "Costs of Parkinson's Disease in a Privately Insured Population." PharmacoEconomics 31 (9): 799-806.

Kaltenboeck, A., S. J. Johnson, M. R. Davis, H. G. Birnbaum, C. A. Carroll, M. L. Tarrants, and A. D. Siderowf. 2012. "Direct Costs and Survival of Medicare Beneficiaries with Early and Advanced Parkinson's Disease.” Parkinsonism \& Related Disorders 18 (4): 321-6.

Kowal, S. L., T. M. Dall, R. Chakrabarti, M. V. Storm, and A. Jain. 2013. "The Current and Projected Economic Burden of Parkinson's Disease in the United States." Movement Disorders 28 (3): 311-8.

Lakdawalla, D., M. R. Eber, F. M. Forma, J. Sullivan, P. C. Michaud, L. A. Bradley, and D. P. Goldman. 2013. "Measuring the Value of Better Diabetes Management." American Journal of Managed Care 19 (2): E11. 
Lakdawalla, D., D. P. Goldman, J. Bhattacharya, M. D. Hurd, G. F. Joyce, and C. W. Panis. 2003. "Forecasting the Nursing Home Population." Medical Care 41 (1): 8-20.

Leidy, N. K. 1994. "Functional Status and the Forward Progress of Merry-Go-Rounds: toward a Coherent Analytical Framework.” Nursing Research 43 (4): 196-202, https://psycnet.apa. org/doi/10.1097/00006199-199407000-00002.

National Institute of Neurological Disorders and Stroke. 2015. “Parkinson's Disease: Challenges, Progress, and promise." National Institute of Neurological Disorder and Stroke, National Institutes of Health. Also available at https://www.ninds.nih.gov/disorders/parkinsons_ disease/parkinsons_research.htm (accessed July 1).

Neumann, P. J., J. T. Cohen, and M. C. Weinstein. 2014. "Updating Cost-Effectiveness - The Curious Resilience of the \$50,000-Per-QALY Threshold." New England Journal of Medicine 371 (9): 796-7.

Olanow, C. W., K. Kieburtz, P. O. Alberto, J. Espay, D. G. Standaert, H. H. Fernandez, A. Vanagunas, A. A. Othman, K. L. Widnell, W. Z. Robieson, Y. Pritchett, K. Chatamra, J. Benesh, R. A Lenz, and A. Antonini. 2014. "Continuous Intrajejunal Infusion of Levodopa-Carbidopa Intestinal Gel for Patients with Advanced Parkinson's Disease: a Randomised, Controlled, DoubleBlind, Double-Dummy Study." The Lancet Neurology 13 (2): 141-9.

PRECISIONheor. 2019. Technical Documentation: The Health Economic Medical Innovation Simulation - HRS Version. Also available at https://www.PRECISIONheor.com/services/ \#anchor3 (accessed May 30).

Pringsheim, T., N. Jette, A. Frolkis, and T. D. Steeves. 2014. "The Prevalence of Parkinson's Disease: A Systematic Review and Meta-Analysis.” Movement Disorders 29 (13): 1583-90.

Shih, T. M., K. R. Sail, Y. J. Jalundhwala, J. Sullivan, E. van Eijndhoven, C. Zadikoff, T. S. Marshall, and D. N. Lakdawalla. 2020. "The Effect of Functional Status Impairment on Nursing Home Admission Risk Among Patients with Advanced Parkinson's Disease.” Journal of Medical Economics 23 (3): 297-307.

Soh, S. E., M. E. Morris, and J. L. McGinley. 2011. "Determinants of Health-Related Quality of Life in Parkinson's Disease: A Systematic Review.” Parkinsonism \& Related Disorders 17 (1): 1-9.

Standaert, D. G., H. H. Fernandez, P. Odin, R. A. Hauser, A. J. Espay, M. Steiger, S. Chouinard, O. Suchowersky, E. Yakupov, E. P. Merikle, W. Z. Robieson, K. Chatamra, and J. Benesh. 2013. "A Long-Term, Open-Label Study of Levodopa-Carbidopa Intestinal Gel in Advanced Parkinson's Disease Patients: Functional and Health-Related Quality-Of-Life Endpoints.” Journal of Neurological Sciences 333: e123-4.

Truven Health Analytics Microdex Solutions. 2016. RedBook Online. Greenwood Village. CO: Truven Health Analytics. .

Nuys, V., A. K. Karen, H. Phatak, U. Iloeje, J. Sullivan, D. N. Lakdawalla, E. Vasudeva, and W. Weintraub. 2014. "Abstract 257: A Long-Term Comparison of Clinical and Economic Outcomes with Novel Oral Anti- Coagulants." Circulation: Cardiovascular Quality and Outcomes 7 (Suppl 1): A257.

van Reenen, M., and B. Janssen. 2015. “EQ-5D-5L User guide.” EuroQol Research Foundation. Also available at https://www.euroqol.org/fileadmin/user_upload/Documenten/PDF/Folders_ Flyers/EQ-5D-5L_UserGuide_2015.pdf (accessed October 4).

Viscusi, W. K., and J. E. Aldy. 2003. "The Value of a Statistical Life: A Critical Review of Market Estimates throughout the World." Journal of Risk and Uncertainty 27 (1): 5-76. 
World Health Organization. 2007. "Neurological Disorders: Public Health challenges.” World Health Organization. Also available at https://www.who.int/mental_health/neurology/ flyer_neurological_report.pdf?ua=1 (accessed October 4).

Supplementary Material: The online version of this article offers supplementary material (https://doi.org/10.1515/fhep-2019-0021). 